\title{
BOUNDING TANGENCIES OF SECTIONS ON ELLIPTIC SURFACES
}

\author{
DOUGLAS ULMER AND GIANCARLO URZÚA
}

\begin{abstract}
Aвstract. Given an elliptic surface $\mathcal{E} \rightarrow \mathcal{C}$ over a field $k$ of characteristic zero equipped with zero section $O$ and another section $P$ of infinite order, we give a simple and explicit upper bound on the number of points where $O$ is tangent to a multiple of $P$.
\end{abstract}

\section{INTRODUCTION}

Let $k=\mathbb{C}$ be the complex numbers 1 , let $\mathcal{C}$ be an irreducible, smooth, projective curve of genus $g$ over $k$, and let $\pi: \mathcal{E} \rightarrow \mathcal{C}$ be a Jacobian elliptic surface over $\mathcal{C}$, i.e., an elliptic surface equipped with a section of $\pi$ denoted $O: \mathcal{C} \rightarrow \mathcal{E}$ which will play the role of a zero section. Let $P: \mathcal{C} \rightarrow \mathcal{E}$ be another section of $\pi$ which is of infinite order in the group law with $O$ as origin.

Write $\mathcal{E}[n]$ for the union of the points of order $n$ in each fiber of $\pi$. It is known that $\mathcal{E}[n]$ is a smooth, locally closed subset of $\mathcal{E}$ which is quasi-finite over $\mathcal{C}$ of generic degree $n^{2}$ (See [UU19, Sections 2.1 and 2.2] for more details.) In [UU19], we proved that the set

$$
\begin{aligned}
T_{\text {tor }} & :=\bigcup_{n>0}\{t \in \mathcal{C} \mid n P \text { is tangent to } O \text { over } t\} \\
& =\bigcup_{n>0}\{t \in \mathcal{C} \mid P \text { is tangent to } \mathcal{E}[n] \text { over } t\}
\end{aligned}
$$

is finite. Our goal in this paper is to give an explicit upper bound for $\left|T_{t o r}\right|$, the cardinality of $T_{t o r}$.

We say that $\mathcal{E}$ is constant if there is an elliptic curve $E$ over $k$ such that $\mathcal{E} \cong \mathcal{C} \times{ }_{k} E$ and $\pi$ is the projection to $\mathcal{C}$. If $\mathcal{E}$ is constant, we say $P$ is constant if there is point $p \in E$ such that $P(t)=(t, p)$ for all $t \in \mathcal{C}$. Let $\delta$ be the number of singular fibers of $\pi: \mathcal{E} \rightarrow \mathcal{C}$, let $\omega=O^{*}\left(\Omega_{\mathcal{E} / \mathcal{C}}^{1}\right)$, and let $d=\operatorname{deg}(\omega)$. Since the discriminant of a Weierstrass model defines a non-vanishing section of $\omega^{\otimes 12}$, we have $d \geq 0$.

Theorem 1.1. Suppose that $\mathcal{E}$ is not constant, or that $\mathcal{E}$ is constant and $P$ is not constant. Then

$$
\left|T_{\text {tor }}\right| \leq 2 g-2-d+\delta .
$$

This is proved as Corollary 7.2 below. In fact, we will prove a more precise result (Theorem7.1) which gives an exact formula for the cardinality, with multiplicities, of a more general set of tangencies.

Date: May 27, 2020.

2010 Mathematics Subject Classification. Primary 14J27; Secondary 11B39, 14 J29.

${ }^{1}$ We will work over $\mathbb{C}$ for simplicity. By a standard reduction given in [UU19], our results also hold when $k$ is any field of characteristic zero. 
1.2. The constant case. The constant case of our result is very transparent and gives a hint of how to proceed in general, so we discuss it here. Suppose that $\mathcal{E} \cong \mathcal{C} \times E$ is constant. Then a section $P: \mathcal{C} \rightarrow \mathcal{E}$ may be identified with a morphism $f: \mathcal{C} \rightarrow E$, and $P$ is constant if and only if $f$ is constant. We assume that $P$ is non-constant. The torsion subset $\mathcal{E}[n]$ consists of the $n^{2}$ constant sections $\mathcal{C} \times\{p\}$ where $p$ is an $n$-torsion point of $E$. It is of interest to consider tangencies with general constant sections $\mathcal{C} \times\{p\}$ for any $p \in E$. Let

$$
T_{\text {const }}:=\bigcup_{p \in E}\{t \in \mathcal{C} \mid P \text { is tangent to } \mathcal{C} \times\{p\} \text { over } t\}
$$

i.e., the set of points of $\mathcal{C}$ where $P$ is tangent to a constant section. Obviously $T_{\text {tor }} \subset T_{\text {const }}$.

To take into account multiplicities, suppose $P(t)=(t, p)$ and let $I(P, t)$ be the intersection number of $\mathcal{C} \times\{p\}$ and $P$ at $(t, p)$. By definition, $I(P, t) \geq 1$ and it is $\geq 2$ if and only if $P$ is tangent to $\mathcal{C} \times\{p\}$ over $t$. On the other hand, it is clear that $I(P, t)$ is $e_{t}(f)$, the ramification index of $f$ at $t$.

Let $\eta_{P}$ be the pull-back under $f$ of a non-zero invariant differential on $E$. Since $f$ is nonconstant, $\eta_{P}$ is a non-zero section of $\Omega_{\mathcal{C}}^{1}$, and the order of vanishing of $\eta_{P}$ at $t$ is

$$
\operatorname{ord}_{t}\left(\eta_{P}\right)=e_{f}(t)-1=I(P, t)-1 \text {. }
$$

Thus we have

$$
\left|T_{\text {tor }}\right| \leq\left|T_{\text {const }}\right| \leq \sum_{t \in \mathcal{C}}(I(P, t)-1)=\sum_{t \in \mathcal{C}} \operatorname{ord}_{t}\left(\eta_{P}\right)=2 g-2 .
$$

Since $d=\delta=0$ when $\mathcal{E}$ is constant, this proves the Theorem 1.1 in the constant case.

1.3. Sketch of the general case. In the general case, we will define a "Betti foliation" on an open subset of $\mathcal{E}$ which generalizes the foliation of $\mathcal{C} \times E$ by the leaves $\mathcal{C} \times\{p\}$ and which has the subsets $\mathcal{E}[n]$ among its closed leaves. This leads to a set of tangencies $T_{B e t t i} \subset \mathcal{C}$ with $T_{\text {tor }} \subset T_{\text {Betti }}$ and intersection multiplicities $I(P, t)$ which measure the order of contact between $P$ and the Betti foliation. We will also define a certain twisted real-analytic 1-form $\eta_{P}$ on an open subset of $\mathcal{C}$ whose local indices $J\left(\eta_{P}, t\right)$ satisfy $J\left(\eta_{P}, t\right)=I(P, t)-1$ at all places $t$ of good reduction. Summing over all points of $\mathcal{C}$ will lead to a formula

$$
\sum_{t \in \mathcal{C}}(I(P, t)-1)=\sum_{t \in \mathcal{C}} J\left(\eta_{P}, t\right)=2 g-2-d,
$$

and taking into account what happens at the bad fibers leads to the upper bound

$$
\left|T_{\text {tor }}\right| \leq\left|T_{\text {Betti }}\right| \leq 2 g-2-d+\delta .
$$

A trivialization essentially equivalent to the Betti foliation was used in the first version of [UU19], and we later adopted the Betti terminology, following [CMZ18]. The form $\eta_{P}$ appears implicitly in the first version of [UU19]. A more general version of it is discussed at some length in [ACZ18, §4], and their account inspired our use of it here to count tangencies. The finiteness of $T_{t o r}$ was proved independently in [CDMZ19]. 
1.4. Plan of the paper. In Section 2 we review certain aspects of Kodaira's construction of $\mathcal{E}$ as an analytic surface. In Sections 3 and 4 , we define the Betti foliation and local intersection numbers $I(P, t)$ measuring the order of contact between a section $P$ and the Betti foliation. In Section 5, we attach to $P$ a real-analytic section $\eta_{P}$ of $\Omega_{\mathcal{C}}^{1} \otimes \omega^{-1}$ over a Zariski open subset of $\mathcal{C}$ and with isolated zeroes, define local indices $J\left(\eta_{P}, t\right)$, and calculate their sum. In Section 6 , we relate the local indices $I(P, t)$ and $J\left(\eta_{P}, t\right)$. This leads to the proof, in Section 7 of the main theorem. Finally, in Section 8 we give examples illustrating edges cases and the sharpness of the main theorem, and we give an application to heights of integral points on elliptic curves over function fields.

1.5. Acknowledgements. The first-named author thanks the Simons Foundation for partial support in the form of Collaboration Grant 359573 and Doug Pickrell for a pointer to the topology literature. The second-named author thanks FONDECYT for support from grant 1190066. Both authors thank Brian Lawrence for drawing their attention to [ACZ18] and an anonymous referee for corrections and for suggesting the application to bounding heights.

\section{2. $\mathcal{E}$ AS AN ANALYTIC SURFACE}

For the rest of the paper, we consider $\mathcal{E}$ as an analytic surface (a 2-dimensional complex manifold) and $\mathcal{C}$ as a Riemann surface. Let $\mathcal{C}^{0} \subset \mathcal{C}$ be the open set over which $\pi$ is smooth and let $\mathcal{E}^{0}=\pi^{-1}\left(\mathcal{C}^{0}\right)$. Let $j: \mathcal{C} \rightarrow \mathbb{P}^{1}$ be the meromorphic function which on $\mathcal{C}^{0}$ sends $t$ to the $j$-invariant of $\pi^{-1}(t)$.

Our goal in this section is to review aspects of the analytic description of $\mathcal{E}$ due to Kodaira. In [Kod63, §7], Kodaira attaches to $\mathcal{E}$ a period map from the universal cover of $\mathcal{C}^{0}$ to the upper half plane and a monodromy representation from the fundamental group of $\mathcal{C}^{0}$ to $\mathrm{SL}_{2}(\mathbb{Z})$. We assume the reader is familiar with these invariants. In [Kod63, §8], Kodaira reconstructs $\mathcal{E}$ from this data, and in [Kod63b $\S 11]$, he describes the group law on (a subset of) $\mathcal{E}$ in sheaf theoretic terms. We will use these ideas in the rest of the paper to define a foliation on $\mathcal{E}$, study its intersections with sections of $\mathcal{E}$, and relate them to a certain real-analytic 1 -form.

2.1. Uniformization. We review the well-known construction of $\mathcal{E}^{0}$ as a quotient space. Let $\widetilde{\mathcal{C}^{0}}$ be the universal cover of $\mathcal{C}^{0}$, choose a point $\tilde{b} \in \widetilde{\mathcal{C}^{0}}$, let $b$ be the image of $\tilde{b}$ in $\mathcal{C}^{0}$, and let $\Gamma=\pi_{1}\left(\mathcal{C}^{0}, b\right)$. Let $\mathcal{H}$ denote the upper half plane. Choosing an oriented basis of $H_{1}\left(\pi^{-1}(b), \mathbb{Z}\right)$, we get a period morphism $\tau: \widetilde{\mathcal{C}^{0}} \rightarrow \mathcal{H}$ and a monodromy representation $\rho: \Gamma \rightarrow \mathrm{SL}_{2}(\mathbb{Z})$. We write

$$
\rho(\gamma)=\left(\begin{array}{ll}
a_{\gamma} & b_{\gamma} \\
c_{\gamma} & d_{\gamma}
\end{array}\right)
$$

The period and monodromy data satisfy the following compatibility: if $\gamma \in \Gamma$ and $\tilde{t} \in \widetilde{\mathcal{C}^{0}}$, then

$$
\tau(\gamma \tilde{t})=\rho(\gamma)(\tau(\tilde{t}))
$$

where $\rho(\gamma)$ acts as a linear fractional transformation on $\mathcal{H}$. 
Form the semi-direct product $\Gamma \ltimes \mathbb{Z}^{2}$ by using the monodromy representation and the right action of $\mathrm{SL}_{2}(\mathbb{Z})$ on $\mathbb{Z}^{2}$ :

$$
\begin{aligned}
\left(\gamma_{1}, m_{1}, n_{1}\right)\left(\gamma_{2}, m_{2}, n_{2}\right) & =\left(\gamma_{1} \gamma_{2},\left(m_{1}, n_{1}\right) \gamma_{2}+\left(m_{2}, n_{2}\right)\right) \\
& =\left(\gamma_{1} \gamma_{2}, a_{\gamma_{2}} m_{1}+c_{\gamma_{2}} n_{1}+m_{2}, b_{\gamma_{2}} m_{1}+d_{\gamma_{2}} n_{1}+n_{2}\right) .
\end{aligned}
$$

For $\tilde{t} \in \widetilde{\mathcal{C}^{0}}$ and $\gamma \in \Gamma$, let

$$
f_{\gamma}(\tilde{t})=\left(c_{\gamma} \tau(\tilde{t})+d_{\gamma}\right)^{-1}
$$

One checks that $f$ satisfies the cocycle relation $f_{\gamma_{1} \gamma_{2}}(\tilde{t})=f_{\gamma_{1}}\left(\gamma_{2} \tilde{t}\right) f_{\gamma_{2}}(\tilde{t})$.

Now let $\Gamma \ltimes \mathbb{Z}^{2}$ act on $\widetilde{\mathcal{C}^{0}} \times \mathbb{C}$ by

$$
(\gamma, m, n)(\tilde{t}, w)=\left(\gamma \tilde{t}, f_{\gamma}(\tilde{t})(w+m \tau(\tilde{t})+n)\right) .
$$

This action is properly discontinuous, and we have isomorphisms

$$
\mathcal{E}^{0} \cong\left(\widetilde{\mathcal{C}^{0}} \times \mathbb{C}\right) /\left(\Gamma \ltimes \mathbb{Z}^{2}\right)
$$

and

$$
\mathcal{C}^{0} \cong \widetilde{\mathcal{C}^{0}} / \Gamma
$$

We will also consider the quotient

$$
\mathcal{F}^{0}:=\left(\widetilde{\mathcal{C}^{0}} \times \mathbb{C}\right) / \mathbb{Z}^{2}
$$

With these isomorphisms and definition, we may identify the diagram of complex manifolds

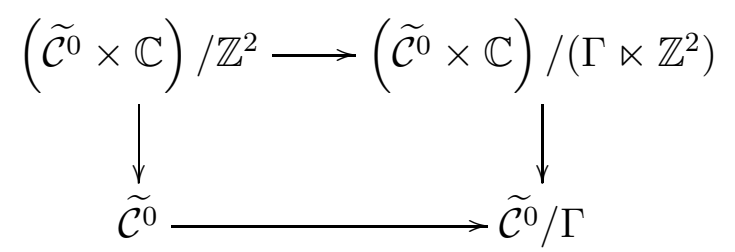

with the Cartesian diagram

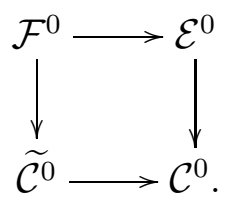

In the introduction, we defined $\omega$ as the line bundle $O^{*}\left(\Omega_{\mathcal{E} / \mathcal{C}}^{1}\right)$. Let $\omega^{-1}$ be the dual line bundle. It is clear from the definitions in this section that a section of $\omega^{-1}$ over an open set $U \subset \mathcal{C}^{0}$ can be identified with a function $w: \tilde{U} \rightarrow \mathbb{C}$ where $\tilde{U}$ is the inverse image of $U$ in $\widetilde{\mathcal{C}^{0}}$ and $w$ satisfies

$$
w(\gamma \tilde{t})=f_{\gamma}(\tilde{t}) w(\tilde{t})
$$


2.2. Global monodromy. We recall three well-known results about the monodromy group $\rho(\Gamma) \subset \mathrm{SL}_{2}(\mathbb{Z}):$

(1) $j: \mathcal{C} \rightarrow \mathbb{P}^{1}$ is non-constant if and only if $\rho(\Gamma)$ is infinite, in which case it has finite index in $\mathrm{SL}_{2}(\mathbb{Z})$.

(2) $j: \mathcal{C} \rightarrow \mathbb{P}^{1}$ is constant if and only if $\rho(\Gamma)$ is finite.

(3) $\mathcal{E}$ is constant if and only if $\rho(\Gamma)$ is trivial.

Indeed, if $j$ is non-constant, the period $\tau$ induces a factorization

$$
\mathcal{C}^{0} \cong \widetilde{\mathcal{C}^{0}} / \Gamma \rightarrow \mathcal{H} / \rho(\Gamma) \rightarrow \mathcal{H} / \mathrm{PSL}_{2}(\mathbb{Z}) \cong \mathbb{A}^{1}
$$

where the composed map $\mathcal{C}^{0} \rightarrow \mathbb{A}^{1}$ is the $j$-invariant. Since $j$ has finite degree, the index of the image of $\Gamma$ in $\operatorname{PSL}_{2}(\mathbb{Z})$ is at most the degree of $j$.

If $j$ is constant, then $\tau$ is constant, and $\mathcal{F}^{0}$ is identified with $\widetilde{\mathcal{C}^{0}} \times E_{b}$ where $E_{b}:=\pi^{-1}(b)$. The action of $\Gamma$ on $\mathcal{F}^{0}$ induces an inclusion $\rho(\Gamma) \subset \operatorname{Aut}\left(E_{b}\right)$. Since the latter has order 2 , 4 , or 6 , this shows that $\rho(\Gamma)$ is finite.

If $\mathcal{E}$ is constant, it is clear that $\rho(\Gamma)$ is trivial. Conversely, if the monodromy is trivial, the argument above shows that $j$ is constant, $\mathcal{F}^{0} \cong \widetilde{\mathcal{C}}^{0} \times E_{b}$, and $\mathcal{E}^{0} \cong \mathcal{F}^{0} / \Gamma \cong \mathcal{C}^{0} \times E_{b}$. Then $\left[\right.$ Kod63, p. 585, $\left.1_{1}\right]$ shows that the isomorphism $\mathcal{E}^{0} \cong \mathcal{C}^{0} \times E_{b}$ extends to an isomorphism $\mathcal{E} \cong$ $\mathcal{C} \times E_{b}$.

We say that $\mathcal{E} \rightarrow \mathcal{C}$ is isotrivial (resp. non-isotrivial) if $j$ is constant (resp. non-constant). Obviously, if $\mathcal{E}$ is constant, it is isotrivial, but not conversely.

2.3. Local invariants. In this section, we recall from [Kod63, §8] the local monodromy, a branch of the period map, and the line bundle $\omega^{-1}$ in a neighborhood of each point $t \in \mathcal{C}$. We use Kodaira's notation $\left(I_{0}, I_{0}^{*}, \ldots\right)$ for the reduction type of each fiber to label the rows of the table at the end of the section.

For each $t \in \mathcal{C}$, let $\Delta_{t}$ be a neighborhood of $t$ biholomorphic to a disk such that $\Delta_{t}^{\prime}=\Delta_{t} \backslash\{t\} \subset$ $\mathcal{C}^{0}$, and let $z$ be a coordinate on $\Delta_{t}$ such that $z=0$ at $t$.

To define the local monodromy, choose a path $p$ from $b$ to a point of $s \in \Delta_{t}^{\prime}$, and let $\gamma$ be a positively oriented loop in $\Delta_{t}^{\prime}$ based at $s$. Then $\rho$ applied to the class of $p^{-1} \gamma p$ is an element $g_{t} \in \rho(\Gamma) \subset \mathrm{SL}_{2}(\mathbb{Z})$ which is well defined up to conjugation by $\rho(\Gamma)$. We say that $g_{t}$ is a generator of the local monodromy at $t$. In the table below, the column "monodromy" gives a representative for the local monodromy for fibers of each type.

If $t \in \mathcal{C}^{0}$, the local monodromy is trivial, and the period map $\tau$ is holomorphic on $\Delta_{t}^{\prime}$ and extends to a holomorphic function on $\Delta_{t}$. If $t \in \mathcal{C} \backslash \mathcal{C}^{0}$, the period map is well-defined on the universal cover $\widetilde{\Delta}_{t}^{\prime}$ of $\Delta_{t}^{\prime}$ and often on a subcover. In the table below, the column "domain" gives a subcover of $\widetilde{\Delta}_{t}^{\prime} \rightarrow \Delta_{t}^{\prime}$ over which the monodromy becomes trivial, and thus over which a branch of $\tau$ becomes a well-defined function. The column "period" describes this function for a suitable choice of a branch of the period map.

We described $\omega^{-1}$ over $\mathcal{C}^{0}$ in the last paragraph of Section 2.1 above. For $t \in \mathcal{C} \backslash \mathcal{C}^{0}$, we may specify $\omega^{-1}$ restricted to $\Delta_{t}$ by giving a section of $\omega^{-1}$ over $\Delta_{t}^{\prime}$ which extends to a generating section over $\Delta_{t}$. Since the monodromy is trivial on the domain, so is the cocycle $f_{\gamma}$, and a section of $\omega^{-1}$ on $\Delta_{t}^{\prime}$ is a function on the domain. The column "generator of $\omega^{-1}$ " describes this function. 


\begin{tabular}{|c|c|c|c|c|}
\hline Fiber & Monodromy & Domain & Period & Generator of $\omega^{-1}$ \\
\hline$I_{0}$ & $\left(\begin{array}{cc}1 & 0 \\
0 & 1\end{array}\right)$ & $z \in \Delta_{t}^{\prime}$ & $\tau=h o l o(z)$ & $w=1$ \\
\hline$I_{b}, b>0$ & $\left(\begin{array}{cc}1 & b \\
0 & 1\end{array}\right)$ & $e^{2 \pi i \zeta}=z$ & $\tau=b \zeta$ & $w=1$ \\
\hline$I_{b}^{*}, b>0$ & $\left(\begin{array}{cc}-1 & -b \\
0 & -1\end{array}\right)$ & $e^{2 \pi i \zeta}=z$ & $\tau=b \zeta$ & $w=e^{\pi i \zeta}$ \\
\hline$I_{0}^{*}$ & $\left(\begin{array}{cc}-1 & 0 \\
0 & -1\end{array}\right)$ & $\zeta^{2}=z$ & $\tau=h o l o(z)$ & $w=\zeta$ \\
\hline$I I$ & $\left(\begin{array}{cc}1 & 1 \\
-1 & 0\end{array}\right)$ & $\zeta^{6}=z$ & $\tau=\frac{\eta-\eta^{2} \zeta^{2 h}}{1-\zeta^{2 h}}, h \equiv 1(\bmod 3)$ & $w=\frac{\zeta}{1-\zeta^{2 h}}$ \\
\hline$I I I$ & $\left(\begin{array}{cc}0 & 1 \\
-1 & 0\end{array}\right)$ & $\zeta^{4}=z$ & $\tau=\frac{i+i \zeta^{2 h}}{1-\zeta^{2 h}}, h \equiv 1(\bmod 2)$ & $w=\frac{\zeta}{1-\zeta^{2 h}}$ \\
\hline$I V$ & $\left(\begin{array}{cc}0 & 1 \\
-1 & -1\end{array}\right)$ & $\zeta^{3}=z$ & $\tau=\frac{\eta-\eta^{2} \zeta^{h}}{1-\zeta^{h}}, h \equiv 2(\bmod 3)$ & $w=\frac{\zeta}{1-\zeta^{h}}$ \\
\hline$I V^{*}$ & $\left(\begin{array}{cc}-1 & -1 \\
1 & 0\end{array}\right)$ & $\zeta^{3}=z$ & $\tau=\frac{\eta-\eta^{2} \zeta^{h}}{1-\zeta^{h}}, h \equiv 1(\bmod 3)$ & $w=\frac{\zeta^{2}}{1-\zeta^{h}}$ \\
\hline$I I I^{*}$ & $\left(\begin{array}{cc}0 & -1 \\
1 & 0\end{array}\right)$ & $\zeta^{4}=z$ & $\tau=\frac{i+i \zeta^{2 h}}{1-\zeta^{2 h}}, h \equiv 1(\bmod 2)$ & $w=\frac{\zeta^{3}}{1-\zeta^{2 h}}$ \\
\hline$I I^{*}$ & $\left(\begin{array}{cc}0 & -1 \\
1 & 1\end{array}\right)$ & $\zeta^{6}=z$ & $\tau=\frac{\eta-\eta^{2} \zeta^{2 h}}{1-\zeta^{2 h}}, h \equiv 2(\bmod 3)$ & $w=\frac{\zeta^{5}}{1-\zeta^{2 h}}$ \\
\hline
\end{tabular}

In the table, we write $\eta$ for $e^{2 \pi i / 3}$ and $h o l o(z)$ for a holomorphic function on $\Delta_{t}^{\prime}$ which extends holomorphically to $\Delta_{t}$.

2.4. Global group structure. Let $\mathcal{E}^{s m}$ be the open subset of $\mathcal{E}$ where $\pi: \mathcal{E} \rightarrow \mathcal{C}$ is smooth, and let $\mathcal{E}^{i d}$ be the union over all $t \in \mathcal{C}$ of the identity component of the fiber of $\mathcal{E}^{s m}$ over $t$. We may view $\mathcal{E}^{i d}$ as the sheaf of abelian groups over $\mathcal{C}$ which assigns to $U \subset \mathcal{C}$ the group of holomorphic sections of $\mathcal{E}^{i d} \rightarrow \mathcal{C}$ over $U$. In [Kod63b, §11], Kodaira gives a description of $\mathcal{E}^{\text {id }}$ in terms of two other sheaves which we now review.

The monodromy representation $\rho$ gives rise to a locally constant sheaf $\mathcal{G}_{0}$ on $\mathcal{C}^{0}$ with stalks $\mathbb{Z}^{2}$. Taking the direct image of $\mathcal{G}_{0}$ along along the inclusion $\mathcal{C}^{0} \subset \mathcal{C}$ yields a sheaf $\mathcal{G}$. Using the description of the local monodromy in the preceding section, we see that the stalk of $\mathcal{G}$ at points of multiplicative reduction $\left(I_{b}, b \geq 1\right)$ is $\mathbb{Z}$, and the stalk at points of additive reduction $\left(I_{b}^{*}, I I\right.$, ...) is 0 .

Using the period morphism $\tau$, we define an inclusion $\mathcal{G} \rightarrow \omega^{-1}$. On $\widetilde{\mathcal{C}}^{0}$ it sends $\mathbb{Z}^{2}$ to $\mathbb{C}$ via $(m, n) \mapsto m \tau(\tilde{t})+n$, and at points of multiplicative reduction it sends $\mathbb{Z} \rightarrow \mathbb{C}$ via $n \mapsto n$.

Kodaira [Kod63b Thm 11.2] showed that there is an exact sequence

$$
0 \rightarrow \mathcal{G} \rightarrow \omega^{-1} \rightarrow \mathcal{E}^{i d} \rightarrow 0
$$

of sheaves of abelian groups on $\mathcal{C}$.

We will use this sequence to work with sections of $\mathcal{E}$ near bad fibers.

\section{The Betti foliation}

In this section, we will define a foliation on $\mathcal{E}^{0}$ which has the torsion multisections $\mathcal{E}^{0} \cap \mathcal{E}[n]$ among its closed leaves. 
3.1. The global Betti foliation. Given $(r, s) \in \mathbb{R}^{2}$, consider the set

$$
\left\{(\tilde{t}, r \tau(\tilde{t})+s) \mid \tilde{t} \in \widetilde{\mathcal{C}}^{0}\right\} \subset \widetilde{\mathcal{C}^{0}} \times \mathbb{C},
$$

and define $\mathcal{F}_{r, s}$ to be its image in $\mathcal{F}^{0}$. Then $\mathcal{F}_{r, s}$ is a section of the projection $\mathcal{F}^{0} \rightarrow \widetilde{\mathcal{C}^{0}}$ which depends only on the class of $(r, s) \in(\mathbb{R} / \mathbb{Z})^{2}$, and we have an isomorphism of real analytic manifolds

$$
\mathcal{F}^{0}=\bigcup_{(r, s) \in(\mathbb{R} / \mathbb{Z})^{2}} \mathcal{F}_{r, s} \cong \widetilde{\mathcal{C}^{0}} \times(\mathbb{R} / \mathbb{Z})^{2}
$$

We define the (global) Betti leaf $\mathcal{G}_{r, s}$ attached to $(r, s) \in(\mathbb{R} / \mathbb{Z})^{2}$ to be the image of $\mathcal{F}_{r, s}$ in $\mathcal{E}^{0} \cong$ $\mathcal{F}^{0} / \Gamma$. (This terminology is inspired by [CMZ18], where $r$ and $s$ are called "Betti coordinates".) The collection of leaves $\mathcal{G}_{r, s}$ gives a foliation of $\mathcal{E}^{0}$ by immersed analytic submanifolds. (In other words, we may give $\mathcal{G}_{r . s}$ the structure of a complex manifold such that the inclusion $\mathcal{G}_{r, s} \rightarrow \mathcal{E}^{0}$ is an immersion. The image is not in general closed, so $\mathcal{G}_{r, s}$ need not be a submanifold in the induced topology.) A straightforward calculation shows that $\mathcal{G}_{r, s}=\mathcal{G}_{r^{\prime}, s^{\prime}}$ if and only if

$$
(r, s)=\left(r^{\prime}, s^{\prime}\right) \rho(\gamma)=\left(a_{\gamma} r^{\prime}+c_{\gamma} s^{\prime}, b_{\gamma} r^{\prime}+d_{\gamma} s^{\prime}\right)
$$

in $(\mathbb{R} / \mathbb{Z})^{2}$ for some $\gamma \in \Gamma$. In particular, the leaves $\mathcal{G}_{r, s}$ are in bijection with the orbits of $\Gamma$ acting on $(\mathbb{R} / \mathbb{Z})^{2}$ from the right.

3.2. The local Betti foliation. We define local Betti leaves as in [UU19]. Let $V \subset \mathcal{C}^{0}$ be nonempty, connected, and simply connected open subset and choose a lifting $V \rightarrow \widetilde{\mathcal{C}^{0}}, t \mapsto \tilde{t}$. Then we get a branch of the period $\tau: V \rightarrow \mathcal{H}, t \mapsto \tau(\tilde{t})$, and we foliate $\pi^{-1}(V) \subset \mathcal{E}^{0}$ by leaves $\mathcal{L}_{r, s}$ where $\mathcal{L}_{r, s}$ is the image of the section of $\mathcal{E}^{0} \rightarrow \mathcal{C}^{0}$ given by

$$
t \mapsto \text { the class of }(\tilde{t}, r \tau(\tilde{t})+s) \in\left(\widetilde{\mathcal{C}^{0}} \times \mathbb{C}\right) /\left(\Gamma \ltimes \mathbb{Z}^{2}\right) \cong \mathcal{E}^{0}
$$

With this definition we have a trivialization

$$
\pi^{-1}(V) \cong V \times(\mathbb{R} / \mathbb{Z})^{2} .
$$

The following relation between the local and global leaves follows immediately from the definitions: for $(r, s) \in(\mathbb{R} / \mathbb{Z})^{2}$,

$$
\pi^{-1}(V) \cap \mathcal{G}_{r, s}=\bigcup_{\left(r^{\prime}, s^{\prime}\right) \in(r, s) \rho(\Gamma)} \mathcal{L}_{r^{\prime}, s^{\prime}}
$$

In other words, over $V$, a global leaf $\mathcal{G}_{r, s}$ decomposes into the disjoint union of local leaves, where the union is indexed by the orbit of the monodromy group on $(\mathbb{R} / \mathbb{Z})^{2}$ through $(r, s)$.

From this we deduce a criterion for a leaf $\mathcal{G}_{r, s}$ to be closed in $\mathcal{E}^{0}$.

\section{Proposition 3.3.}

(1) If $\mathcal{E}$ is isotrivial, every leaf $\mathcal{G}_{r, s}$ is closed.

(2) If $\mathcal{E}$ is non-isotrivial, $\mathcal{G}_{r, s}$ is closed if and only if $(r, s) \in(\mathbb{Q} / \mathbb{Z})^{2}$ if and only if every point of $\mathcal{G}_{r, s}$ is a torsion point in its fiber.

(3) If $\mathcal{E}$ is not constant, then a section $P$ has image lying in a leaf $\mathcal{G}_{r, s}$ if and only if $P$ is a torsion section. 
Proof. From the local description above, it is clear that $\mathcal{G}_{r, s}$ is closed in $\mathcal{E}^{0}$ if the orbit of $\rho(\Gamma)$ through $(r, s)$ is finite. Since $\rho(\Gamma)$ is finite when $\mathcal{E}$ is isotrivial, this establishes part (1).

For part (2), suppose that $\mathcal{E}$ is non-isotrivial. Then as noted in Section 2.2, $\rho(\Gamma)$ has finite index in $\mathrm{SL}_{2}(\mathbb{Z})$. If $(r, s) \in(\mathbb{Q} / \mathbb{Z})^{2}$ it is clear that the orbit through $(r, s)$ is finite and that $\mathcal{G}_{r, s}$ consists of points which are torsion in their fiber. Suppose then that $(r, s) \in(\mathbb{R} / \mathbb{Z})^{2} \backslash(\mathbb{Q} / \mathbb{Z})^{2}$. It is clear that the points of $\mathcal{G}_{r, s}$ are not torsion in their fiber. Since $\rho(\Gamma)$ has finite index in $\mathrm{SL}_{2}(\mathbb{Z})$, there is an integer $b$ such that $\left(\begin{array}{ll}1 & b \\ 0 & 1\end{array}\right) \in \rho(\Gamma)$. If $r \notin \mathbb{Q}$, then the orbit contains

$$
(r, s)\left(\begin{array}{ll}
1 & b \\
0 & 1
\end{array}\right)^{n}=(r, n b r+s)
$$

and thus $\mathcal{G}_{r, s}$ is not closed by Weyl equidistribution. If $s \notin \mathbb{Q}$, a similar argument shows that $\mathcal{G}_{r, s}$ is not closed. This completes the proof of part (2).

For part (3), assume that $\mathcal{E}$ is not constant and that $P$ is a section. If $P$ is torsion, then in every fiber its "Betti coordinates" $(r, s)$ are rational. Since $\mathbb{Q}$ is totally disconnected, these coordinates must be the same in every fiber, so $P$ lies in $\mathcal{G}_{r, s}$ for some rational pair $(r, s)$. Conversely, if $P$ lies in $\mathcal{G}_{r, s}$ then $(r, s)$ must be invariant under the monodromy group $\rho(\Gamma)$. Similarly for the multiples $n P$. But $\mathcal{E}$ is non-constant, and this implies that the monodromy group is non-trivial and either finite or of finite index in $\mathrm{SL}_{2}(\mathbb{Z})$ (as noted in Section 2.2). In both cases, it has elements with only finitely many fixed points on $(\mathbb{R} / \mathbb{Z})^{2}$, so the set $\{n P \mid n \in \mathbb{Z}\}$ is finite, i.e., $P$ is torsion. This completes the proof of part (3).

3.4. Behavior at infinity. We consider the local geometry of Betti leaves near a singular fiber. Suppose $t \in \mathcal{C} \backslash \mathcal{C}^{0}$ and, as in Section 2.3, let $\Delta_{t} \subset \mathcal{C}$ be a neighborhood of $t$ biholomorphic to a disk with $\Delta_{t}^{\prime}:=\Delta_{t} \backslash\{t\} \subset \mathcal{C}^{0}$. Let $V \subset \Delta_{t}^{\prime}$ be a non-empty, connected, and simply connected open set, and define the local monodromy $g_{t} \in \mathrm{SL}_{2}(\mathbb{Z})$ as in Section 2.3 and local Betti leaves $\mathcal{L}_{r, s}$ as in Section 3.2 .

We say that a local leaf $\mathcal{L}_{r, s}$ is an invariant leaf (with respect to $t$ ) if $(r, s) \in(\mathbb{R} / \mathbb{Z})^{2}$ is fixed by $g_{t}$ (acting on the right), and we say it is a vanishing leaf (with respect to $t$ ) if $(r, s)$ is not invariant under $g_{t}$. The latter terminology is motivated by part (4) of the following result.

\section{Proposition 3.5.}

(1) If $\mathcal{L}_{r, s}$ is an invariant leaf, then it extends to a section of $\pi: \mathcal{E} \rightarrow \mathcal{C}$ over $\Delta_{t}$, and this section meets the special fiber $\pi^{-1}(t)$ in a smooth point.

(2) If $\mathcal{E}$ has multiplicative reduction at $t$ (type $\left.I_{b}, b \geq 1\right)$, let $S \cong(\mathbb{Z} / b \mathbb{Z}) \times S^{1}$ be the closure of the set of points of finite order in the special fiber. The invariant leaves extend to sections meeting the special fiber at points of $S$, and every point of $S$ is met by the extension of a unique invariant leaf $\mathcal{L}_{r, s}$.

(3) If $\mathcal{E}$ has additive reduction at $t$ (types $I_{b}^{*}, b \geq 0, I I, I I^{*}, I I I, I I I^{*}, I V, I V^{*}$ ), the invariant leaves extend to sections meeting the special fiber at one of its finitely many torsion points, and each such point is met by the extension of a unique invariant leaf $\mathcal{L}_{r, s}$.

(4) If $\mathcal{L}_{r, s}$ is a vanishing leaf, then it extends to a connected multisection of $\mathcal{E} \rightarrow \mathcal{C}$ over $\Delta_{t}$ of degree $>1$ (possibly infinite), and this multisection meets the special fiber $\pi^{-1}(t)$ in one singular point.

Proof. Suppose $\mathcal{L}_{r, s}$ is an invariant leaf. Then by analytic continuation, $\mathcal{L}_{r, s}$ extends to a section of $\pi$ over $\Delta_{t}^{\prime}$. The closure of of this section in $\pi^{-1}\left(\Delta_{t}\right)$ is proper over $\Delta_{t}$ (since $\pi$ is proper) and 
by invariance of the intersection number, it meets the special fiber with intersection number 1 , and thus must meet it at a smooth point. This establishes part (1).

Now assume that $\mathcal{E}$ has reduction type $I_{1}$ at $t$. Let $\mathcal{X}=\pi^{-1}\left(\Delta_{t}\right)$ and $\mathcal{X}^{\prime}=\pi^{-1}\left(\Delta_{t}^{\prime}\right)$. Then Kodaira showed that

$$
\mathcal{X} \backslash \mathcal{X}^{\prime}=\text { nodal cubic } \cong \mathbb{C}^{\times} \cup\{q\}
$$

where $q$ is the node of the cubic, and that, with a suitable choice of coordinates, $\mathcal{X}^{\prime}$ has the form

$$
\mathcal{X}^{\prime} \cong\left(\Delta_{t}^{\prime} \times \mathbb{C}^{\times}\right) / \mathbb{Z}
$$

where the action of $\mathbb{Z}$ on $\Delta_{t}^{\prime} \times \mathbb{C}^{\times}$is

$$
m \cdot(u, v)=\left(u, u^{m} v\right)
$$

Moreover, there is a holomorphic map

$$
\phi: \Delta_{t} \times \mathbb{C}^{\times} \rightarrow \mathcal{X}
$$

such that $\{t\} \times \mathbb{C}^{\times}$maps biholomorphically to the complement of $q$ in the special fiber, and $\Delta^{\prime} \times \mathbb{C}^{\times} \rightarrow \mathcal{X}^{\prime} \subset \mathcal{X}$ is the natural quotient map.

In terms of a suitable basis, the local monodromy map is

$$
g_{t}=\left(\begin{array}{ll}
1 & 1 \\
0 & 1
\end{array}\right) \text {. }
$$

It is then straightforward to calculate that the invariant leaves are those of the form $\mathcal{L}_{0, s}$ for $s \in \mathbb{R} / \mathbb{Z}$. The corresponding extended section is

$$
u \mapsto \text { the class of }\left(u, e^{2 \pi i s}\right),
$$

these sections specialize to points on the unit circle $S=S^{1} \subset \mathbb{C}^{\times}$, and we get the asserted bijection between the invariant leaves and points on $S$. The establishes the case $b=1$ of part (2).

The case of $I_{b}$ reduction for general $b$ is very similar, with additional notational complexities. In suitable coordinates, the local monodromy is

$$
g_{t}=\left(\begin{array}{ll}
1 & b \\
0 & 1
\end{array}\right)
$$

and the invariant leaves are those of the form $\mathcal{L}_{r, s}$ where $r \in(1 / b) \mathbb{Z} / \mathbb{Z}$ and $s \in \mathbb{R} / \mathbb{Z}$.

The smooth part of $\mathcal{X}=\pi^{-1}\left(\Delta_{t}\right)$ is covered by open subsets as follows: For $i \in \mathbb{Z} / b \mathbb{Z}$, let

$$
W_{i}=W_{i}^{\prime} \cup \mathbb{C}_{i}^{\times}, \quad W_{i}^{\prime}=\left(\Delta_{t}^{\prime} \times \mathbb{C}^{\times}\right) / \mathbb{Z}
$$

where the action of $\mathbb{Z}$ on $\Delta_{t}^{\prime} \times \mathbb{C}^{\times}$is

$$
m \cdot(u, v)=\left(u, u^{b m} v\right) .
$$

For $u \in \Delta_{t}^{\prime}$ and $v \in \mathbb{C}^{\times}$, write $(u, v)_{i}$ for the class of $(u, v)$ in $W_{i}^{\prime}$. Then $\mathcal{X}^{s m}$ is obtained by gluing the $W_{i}$ according to the rule

$$
(u, v)_{i}=\left(u, u^{j-i} v\right)_{j}
$$

for all $u \in \Delta_{t}^{\prime}, v \in \mathbb{C}^{\times}$, and $i, j \in \mathbb{Z} / b \mathbb{Z}$.

The invariant leaf $\mathcal{L}_{i / b, s}$ lies in the open corresponding to $i$ and extends to the section

$$
u \mapsto \text { the class of }\left(u, e^{2 \pi i s}\right)_{i},
$$


and we find that the specializations of extensions of invariant leaves are in bijection with

$$
(1 / b) \mathbb{Z} / \mathbb{Z} \times S^{1} \subset \pi^{-1}(t),
$$

as required. This establishes part (2) in the general case.

For part (3), recall the explicit generators for the local monodromy groups in the table at the end of Section 2.3. Using these, one computes the invariant leaves, which are as follows:

$$
\begin{aligned}
I_{b}^{*}, b \text { odd } & : \mathcal{L}_{0,0}, \mathcal{L}_{1 / 2,1 / 4}, \mathcal{L}_{0,1 / 2}, \mathcal{L}_{1 / 2,3 / 4} \\
I_{b}^{*}, b \text { even } & : \mathcal{L}_{0,0}, \mathcal{L}_{1 / 2,1 / 2}, \mathcal{L}_{0,1 / 2}, \mathcal{L}_{1 / 2,0} \\
I I, I I^{*}: & : \mathcal{L}_{0,0} \\
I I I, I I I^{*}: & : \mathcal{L}_{0,0}, \mathcal{L}_{1 / 2,1 / 2} \\
I V, I V^{*}: & : \mathcal{L}_{0,0}, \mathcal{L}_{1 / 3,2 / 3}, \mathcal{L}_{2 / 3,1 / 3} .
\end{aligned}
$$

The results of Kodaira recalled in Section 2.4 show that for these reduction types, the connected component of the special fiber is isomorphic to the additive group (and so is torsion free), and the group of torsion points on the special fiber is isomorphic to the group of components. It is then straightforward to see that each of the corresponding sections specializes to a torsion point and that all torsion points on the special fiber are met by the extension of a unique invariant leaf.

For part (4), it is clear that analytic continuation of a vanishing leaf $\mathcal{L}_{r, s}$ yields a multisection over $\Delta_{t}^{\prime}$ whose degree is the order of the orbit of the monodromy group through $(r, s)$, which by assumption is $>1$. That its closure in $\pi^{-1}\left(\Delta_{t}\right)$ adds a single point over $t$ which is singular in the special fiber requires a tedious analysis of cases. Since we will not use this result elsewhere in the paper, we omit the details.

\section{Intersections With the Betti foliation}

For the rest of the paper, we assume that $\pi: \mathcal{E} \rightarrow \mathcal{C}$ is non-constant and that $P$ is not torsion. In this section, we will quantify tangencies between $P$ and the Betti foliation in terms of intersection numbers.

4.1. Local intersection numbers. Suppose first that $t \in \mathcal{C}^{0}$, i.e., that $\mathcal{E}$ has good reduction at $t$. Over a neighborhood of $t$, there is a unique local Betti leaf $\mathcal{L}$ passing through $P(t)$. Since $P$ is not torsion, Proposition 3.3(3) implies that this intersection is isolated, i.e., by shrinking the neighborhood, we may assume $P$ and $\mathcal{L}$ meet only over $t$. We define $I(P, t)$ to be the intersection multiplicity of $P$ and $\mathcal{L}$ at $P(t)$. (This is the local intersection number of two holomorphic 1manifolds meeting at an isolated point of a holomorphic 2-manifold. We will make it explicit in terms of the order of vanishing of a holomorphic function below.)

Note that the intersection in question satisfies $I(P, t) \geq 1$, and $I(P, t) \geq 2$ if and only if $P$ is tangent to $\mathcal{L}$ at $t$, i.e., if and only if $t \in T_{\text {Betti }}$.

Now assume that $t \in \mathcal{C} \backslash \mathcal{C}^{0}$. Let $S \subset \pi^{-1}(t)$ be the closure of the set of torsion points in the special fiber. As noted in Proposition 3.5, $S \cong(\mathbb{Z} / b \mathbb{Z}) \times S^{1}$ if $\mathcal{E}$ has reduction type $I_{b}$ at $t$, and it is a finite group in the other cases. If $P(t) \notin S$, we define $I(P, t)=0$. If $P(t) \in S$, then by Proposition 3.5, there is a unique invariant local leaf $\mathcal{L}$ extending over a neighborhood of $t$ and meeting $P$ over $t$. We define $I(P, t)$ to be the intersection number of $P$ and this extended leaf at $t$. 
Lemma 4.2. For all integers $n>0$ and all points $t \in \mathcal{C}$,

$$
I(P, t)=I(n P, t) .
$$

Proof. Indeed, since the multiplication by $n$ map $\mathcal{E}^{s m} \rightarrow \mathcal{E}^{s m}$ is étale, for every $(r, s) \in(\mathbb{R} / \mathbb{Z})^{2}$, the intersection number of $P$ with the local leaf $\mathcal{L}_{r, s}$ at $t$ is the same as the intersection number of $n P$ with $\mathcal{L}_{n r, n s}$ at $t$.

4.3. Explicit intersection numbers. In this section, we make the intersection number $I(P, t)$ more explicit by using the exact sequence (2.2).

By Lemma 4.2 we may replace $P$ with a multiple and thereby assume that $P$ passes through the identity component of each fiber.

Fix $t \in \mathcal{C}$ and choose a small enough neighborhood $\Delta_{t}$ of $t$ in $\mathcal{C}$ such that the restricted section $P: \Delta_{t} \rightarrow \mathcal{E}$ lifts to a section of $\omega^{-1}$ over $\Delta_{t}$ and such that $\omega^{-1}$ is trivial over $\Delta_{t}$. Let $z$ be a coordinate on $\Delta_{t}$ such that $t$ corresponds to $z=0$. We may then identify $P$ with a product $w=h w_{0}$ where $h$ is a holomorphic function on $\Delta_{t}$ and $w_{0}$ is a generating section of $\omega^{-1}$ over $\Delta_{t}$ as specified in the table at the end of Section 2.3 .

The local multiplicity $I(P, t)$ is by definition the intersection number of $P$ and an invariant local Betti leaf $\mathcal{L}_{r, s}$. Since the leaf is invariant, the map $z \mapsto r \tau(z)+s$ defines a section of $\omega^{-1}$ over $\Delta_{t}$, and the intersection multiplicity is the same as the intersection number between the graphs of the functions $z \mapsto h(z)$ and $z \mapsto(r \tau(z)+s) / w_{0}$

If $t \in \mathcal{C}^{0}$, then $w_{0}=1$. If $h(t)=r \tau(t)+s, I(P, t)$ is the intersection number between the graph of $z \mapsto h(z)$ and the graph of $z \mapsto r \tau(z)+s$. Therefore,

$$
I(P, t)=\operatorname{ord}_{z=0}(h(z)-r \tau(z)-s) .
$$

If $\mathcal{E}$ has multiplicative reduction $\left(I_{b}, b>0\right)$ at $t$, then $w_{0}=1$ and $I(P, t)=0$ if $h(t) \notin \mathbb{R}$. If $h(t)=s \in \mathbb{R}$, then $I(P, t)$ is the intersection number between the graph of $z \mapsto h(z)$ and the graph of the constant function $z \mapsto s$. Therefore,

$$
I(P, t)= \begin{cases}0 & \text { if } h(t) \notin \mathbb{R} \\ \operatorname{ord}_{z=0}(h(z)-s) & \text { if } h(t)=s \in \mathbb{R} .\end{cases}
$$

If $\mathcal{E}$ has additive reduction at $t$ (types $\left.I_{b}^{*}, I I, \ldots\right)$, then $I(P, t)=0$ unless $h(t)=0$, and if $h(t)=0$, then $I(P, t)$ is the intersection number between the graph of $z \mapsto h(z)$ and the graph of $z \mapsto 0$. Therefore,

$$
I(P, t)=\operatorname{ord}_{z=0}(h(z))
$$

\section{A ReAl ANAlytic 1-Form}

In this section, we review a connection between local and global degrees of smooth sections of a line bundle. We then construct a real analytic 1-form whose zeroes will turn out to control tangencies between a section $P$ and the Betti foliation.

5.1. Local and global indices. The number of zeroes and poles of a meromorphic section of a line bundle (counted with multiplicities) is the degree of the line bundle. This familiar result from basic algebraic geometry is in fact purely topological. In this section, we state and sketch the proof of the result in the smooth category. Our Proposition 5.1.4 is in substance equivalent to 
[BT82, Thm. 11.17], but the language there is rather different than ours, so for the convenience of the reader, we review the main lines of the argument adapted to our situation.

5.1.1. Winding numbers. Let $\Delta$ be the unit disk in $\mathbb{C}$, and let $\Delta^{\prime}=\Delta \backslash\{0\}$. Suppose that $f$ is a smooth, nowhere vanishing, complex-valued function on $\Delta^{\prime}$. We define the winding number of $f$ to be

$$
W(f):=\frac{1}{2 \pi i} \oint d \log f
$$

where the path of integration is any positively oriented loop around 0. Equivalently

$$
W(f)=\frac{1}{2 \pi i} \int_{0}^{1} \frac{g^{\prime}(t)}{g(t)} d t
$$

where $g(t)=f\left(r e^{2 \pi i t}\right)$ for some $0<r<1$.

The following properties of $W(f)$ are well known. See, for example, [Ful95, Ch. 3].

(1) $W(f)$ is an integer and is independent of the choice of path of integration.

(2) If $f$ extends to a smooth nowhere vanishing function on $\Delta$, then $W(f)=0$.

(3) $W\left(f_{1} f_{2}\right)=W\left(f_{1}\right)+W\left(f_{2}\right)$.

(4) If $f$ is the restriction of a meromorphic function on $\Delta$, then $W(f)=\operatorname{ord}_{z=0} f(z)$.

(5) If $F(\sigma, z)$ is a smooth, nowhere vanishing function on $[0,1] \times \Delta^{\prime}$ and $f_{\sigma}(z)=F(\sigma, z)$, then $W\left(f_{0}\right)=W\left(f_{1}\right)$.

The following is essentially the "dog on a leash" theorem, see [Ful95, Thm 3.11].

\section{Lemma 5.1.2.}

(1) Suppose that $f_{1}$ and $f_{2}$ are smooth functions on the punctured disk $\Delta^{\prime}$, and let $f=f_{1}-f_{2}$. Suppose also that there exist real numbers $m_{1}<m_{2}$ and positive real numbers $C_{1}$, and $C_{2}$ such that

$$
\left|f_{1}(z)\right| \geq C_{1}|z|^{m_{1}}
$$

and

$$
\left|f_{2}(z)\right| \leq C_{2}|z|^{m_{2}}
$$

for all $\in \Delta^{\prime}$. Then $W(f)=W\left(f_{1}\right)$.

(2) The same conclusion holds when $m_{1}=m_{2}$ provided that $C_{1}>C_{2}$.

Proof. Define $F$ on $[0,1] \times \Delta^{\prime}$ by $F(\sigma, z)=f_{1}(z)-\sigma f_{2}(z)$, so that $F(1, z)=f(z)$ and $F(0, z)=$ $f_{1}(z)$. The displayed inequalities show that $F(\sigma, z) \neq 0$ for all sufficiently small $z$, so we may shrink $\Delta^{\prime}$ and have that $F(\sigma, z)$ is nowhere vanishing on $[0,1] \times \Delta^{\prime}$. The winding numbers $W(f)$ and $W\left(f_{1}\right)$ are then well defined, and property (5) of winding numbers shows that $W(f)=$ $W\left(f_{1}\right)$.

5.1.3. Local indices. Let $L$ be a holomorphic line bundle on $\mathcal{C}$ and suppose that $s$ is a smooth, nowhere vanishing section of $L$ over an open subset of the form $U=\mathcal{C} \backslash\left\{t_{1}, \ldots, t_{m}\right\}$. We define a local index $J(s, t)$ for all $t \in \mathcal{C}$ as follows: Given $t$, choose a neighborhood $U_{t}$ of $t$ diffeomorphic to a disk and such that $s$ is defined and non-zero on $U_{t}^{\prime}:=U_{t} \backslash\{t\}$. Choose a trivializing section $s_{t}$ of $L$ (as a complex line bundle) over $U_{t}$, and write $s=f(z) s_{t}$ for $z \in U_{t}^{\prime}$. Then

$$
J(s, t):=W(f)
$$


where we identify $f$ with a function on $\Delta^{\prime}$ via a diffeomorphism $\Delta \cong U_{t}$ sending 0 to $t$. The properties of $W$ recalled above imply that $J(s, t)$ is an integer and is independent of the various choices. The also imply that if $s$ is a meromorphic section of $L$ near $t$, then $J(s, t)$ is exactly the order of zero or pole of $s$ at $t$ in the usual sense.

The following global result generalizes the statement that the sum of the orders of zero or pole of a meromorphic section of a line bundle is the degree of the line bundle. Recall that $H^{2}(\mathcal{C}, \mathbb{Z})$ is canonically isomorphic to $\mathbb{Z}$. We $\operatorname{define} \operatorname{deg}(L)$ to be the first Chern class $c_{1}(L) \in H^{2}(\mathcal{C}, \mathbb{Z})=\mathbb{Z}$.

Proposition 5.1.4. Suppose $s$ is a smooth, nowhere vanishing section of $L$ over $U=\mathcal{C} \backslash\left\{t_{1}, \ldots, t_{m}\right\}$. Then

$$
\sum_{t \in \mathcal{C}} J(s, t)=\sum_{i=1}^{m} J\left(s, t_{i}\right)=\operatorname{deg}(L) .
$$

Proof. Property (2) of winding numbers recalled above implies that $J(s, t)=0$ unless $t$ is in $\left\{t_{1}, \ldots, t_{m}\right\}$, so the sum over $t \in \mathcal{C}$ is well defined and equal to the sum over the $t_{i}$. To prove the equality with the degree of $L$ we will compare Čech and de Rham cohomologies.

For $i=1, \ldots, m$, let $U_{i}$ be a neighborhood of $t_{i}$ diffeomorphic to the disk $\Delta$ with 0 corresponding to $t_{i}$ and such that the closures of the $U_{i}$ in $\mathcal{C}$ are disjoint. Choose simply connected open sets $U_{m+1}, \ldots, U_{n} \subset U$ such that $U_{1}, \ldots, U_{n}$ covers $\mathcal{C}$ and such that $U_{i j}:=U_{i} \cap U_{j}$ is simply connected for all pairs of indices $1 \leq i, j \leq n$. For $i=1, \ldots, m$, choose generating sections $s_{i}$ of $L$ over $U_{i}$, and for $i=m+1, \ldots, n$, let $s_{i}$ be the restriction of $s$ to $U_{i}$. Then there are smooth, nowhere vanishing functions $g_{i j}$ defined on $U_{i j}$ by

$$
s_{i}=g_{i j} s_{j},
$$

and the $g_{i j}$ form a 1-cocycle with values in $\mathcal{A}^{\times}$, the sheaf of nowhere vanishing smooth functions on $\mathcal{C}$. The class of this cocycle in Čech cohomology is $[L] \in H^{1}\left(\mathcal{C}, \mathcal{A}^{\times}\right)$.

We have an exact sequence

$$
0 \rightarrow \mathbb{Z} \rightarrow \mathcal{A} \rightarrow \mathcal{A}^{\times} \rightarrow 0
$$

where $\mathcal{A}$ is the sheaf of smooth functions on $\mathcal{C}$ and the map $\mathcal{A} \rightarrow \mathcal{A}^{\times}$is $f \mapsto e^{2 \pi i f}$. Taking the coboundary of $[L]$ in the long exact sequence of cohomology, we find that $c_{1}(L) \in H^{2}(\mathcal{C}, \mathbb{Z})$ is represented by the 2-cocycle

$$
\eta_{i j k}=\frac{1}{2 \pi i}\left(\log g_{i j}-\log g_{i k}+\log g_{j k}\right) .
$$

Next, we write down a 2-form representing the image of $[L]$ under

$$
H^{2}(\mathcal{C}, \mathbb{Z}) \rightarrow H^{2}(\mathcal{C}, \mathbb{C}) \cong H_{d R}^{2}(\mathcal{C}) \otimes \mathbb{C}
$$

Since $\eta_{i j k}$ is $\mathbb{Z}$-valued, we have $d \eta_{i j k}=0$. This implies that

$$
h_{i j}=\frac{1}{2 \pi i} d \log g_{i j}
$$

is a 1-cocycle with values in $\mathcal{A}^{1}$, the sheaf of smooth 1 -forms on $\mathcal{C}$.

Now choose a partition of unity $\rho_{i}$ subordinate to the cover $U_{i}$ of $\mathcal{C}$. Shrinking $U_{i}$ for $i=$ $m+1, \ldots, n$ if necessary, we may assume that for $i=1, \ldots, m$, there are closed disks of positive radius $K_{i, 1} \subset K_{i, 2} \subset U_{i}$ such that $\rho_{i}$ is identically 1 on $K_{i, 1}$ and identically zero on the complement of $K_{i, 2}$. 
Setting

$$
\theta_{i}=\frac{1}{2 \pi i} \sum_{\ell=1}^{n} \rho_{\ell} d \log g_{i \ell} \in \mathcal{A}^{1}\left(U_{i}\right)
$$

we see that $\theta_{i}-\theta_{j}=h_{i j}$. Since $h_{i j}$ is $d$-closed for all $i j$, we find that $d \theta_{i}=d \theta_{j}$ on $U_{i j}$ and so we may define a global 2-form $\Omega$ on $\mathcal{C}$ by requiring that

$$
\begin{aligned}
\Omega & =-d \theta_{i} \\
& =\frac{-1}{2 \pi i} \sum_{\ell=1}^{n} d \rho_{\ell} d \log g_{i \ell}
\end{aligned}
$$

on $U_{i}$.

It follows from the "generalized Mayer-Vietoris principle" [BT82, §8] (also known fondly to some as the "Čech-de Rham shuffle"), that $\Omega$ represents the class of $L$ in de Rham cohomology. More formally

$$
c_{1}(L)=\int_{\mathcal{C}} \Omega
$$

(The point is that the $\mathbb{Z}$-valued 2-cocycle $\eta_{i j k}$ and the $\mathcal{A}^{2}$-valued 0 -cocycle $\Omega$ represent the same class in the cohomology of the total complex of the Čech-deRham double complex because, by construction, they differ by a coboundary.)

To finish the proof, we will relate the displayed integral to winding numbers. Let $U_{i}^{\prime}=U_{i} \backslash\left\{t_{i}\right\}$, and let $g_{i} \in \mathcal{A}^{\times}\left(U_{i}^{\prime}\right)$ be defined by

$$
s_{i}=g_{i} s
$$

Then examining the definitions shows that

$$
\begin{aligned}
g_{i j}=1 & \text { if } i, j>m \\
g_{i j}=g_{i} & \text { if } i \leq m \text { and } j>m \\
U_{i} \cap U_{j}=\emptyset & \text { if } i, j \leq m .
\end{aligned}
$$

It follows that $\Omega$ vanishes identically on the complement of $\cup_{i=1}^{m} U_{i}$, and so

$$
\operatorname{deg}(L)=\sum_{i=1}^{m} \int_{U_{i}} \Omega .
$$

On $U_{i}$ we have

$$
\begin{aligned}
\Omega_{\mid U_{i}} & =\frac{-1}{2 \pi i} \sum_{\ell=m+1}^{n} d \rho_{\ell} d \log g_{i} \\
& =\frac{1}{2 \pi i} d \rho_{i} d \log g_{i} .
\end{aligned}
$$

Now $d \rho_{i}$ is identically zero on $K_{i, 1}$ and on the complement of $K_{i, 2}$, so we have

$$
\begin{aligned}
\int_{U_{i}} \Omega & =\frac{1}{2 \pi i} \int_{K_{i, 2} \backslash K_{i, 1}} d \rho_{i} d \log g_{i} \\
& =\frac{1}{2 \pi i} \int_{\partial K_{i, 2}} \rho_{i} d \log g_{i}-\frac{1}{2 \pi i} \int_{\partial K_{i, 1}} \rho_{i} d \log g_{i}
\end{aligned}
$$


by Stokes' theorem. Since $\rho_{i}$ vanishes on $\partial K_{i, 2}$ and is 1 on $\partial K_{i, 1}$, we find

$$
\int_{U_{i}} \Omega=-W\left(g_{i}\right)=W\left(g_{i}^{-1}\right)=J\left(s, t_{i}\right)
$$

where the last equality follows from the definition of $J$. Combining this with Equation (5.1), we find that

$$
\operatorname{deg}(L)=\sum_{i=1}^{m} J\left(s, t_{i}\right)
$$

as desired. This completes the proof of the proposition.

5.2. Constructing $\eta$. We use the uniformization of Section 2.1, Let $w$ be the standard coordinate on $\mathbb{C}$ and recall the period function $\tau: \widetilde{\mathcal{C}^{0}} \rightarrow \mathcal{H}$. Consider the real-analytic 1 -form on $\widetilde{\mathcal{C}^{0}} \times \mathbb{C}$ given by

$$
\tilde{\eta}=d w-\frac{\operatorname{Im} w}{\operatorname{Im} \tau} d \tau
$$

Under the action of $\Gamma \ltimes \mathbb{Z}^{2}$, straightforward calculation shows that

$$
(i d, m, n)^{*}(\tilde{\eta})=\tilde{\eta}
$$

and

$$
(\gamma, 0,0)^{*}(\tilde{\eta})=f_{\gamma} \tilde{\eta}=\frac{\tilde{\eta}}{c_{\gamma} \tau+d_{\gamma}}
$$

where as usual $\rho(\gamma)=\left(\begin{array}{ll}a_{\gamma} & b_{\gamma} \\ c_{\gamma} & d_{\gamma}\end{array}\right)$. These formulas show that $\tilde{\eta}$ descends to a real-analytic section $\eta$ of

$$
\Omega_{\mathcal{E}^{0}}^{1} \otimes \pi^{*}(\omega)^{-1}=\Omega_{\mathcal{E}^{0}}^{1} \otimes\left(\Omega_{\mathcal{E}^{0} / \mathcal{C}^{0}}^{1}\right)^{-1} .
$$

It is immediate from the definition of the Betti foliation in terms of the uniformization $\widetilde{\mathcal{C}^{0}} \times \mathbb{C} \rightarrow$ $\mathcal{E}^{0}$ that at every point $x \in \mathcal{E}^{0}$, the kernel of $\eta$ as a functional on the holomorphic tangent space of $\mathcal{E}^{0}$ at $x$ is precisely the tangent space to the leaf of the Betti foliation passing through $x$. We will thus be able to use $\eta$ to quantify the tangencies between sections $P$ and the Betti foliation.

5.3. Definition of $\eta_{P}$. Now assume that $P$ is a non-torsion section of $\mathcal{E} \rightarrow \mathcal{C}$ and recall that the latter is assumed to be non-constant. Let $\eta_{P}:=P^{*}(\eta)$. This is a real analytic section of $\Omega_{\mathcal{C}}^{1} \otimes \omega^{-1}$ over $\mathcal{C}^{0}$. Since the kernel of $\eta$ at a point of $\mathcal{E}^{0}$ is the tangent space to the leaf of the Betti foliation through that point, we see that $\eta_{P}$ vanishes at a point of $\mathcal{C}^{0}$ if and only if that point lies in $T_{\text {Bett } i} \cap \mathcal{C}^{0}$. Since $T_{\text {Betti }}$ is finite by [UU19, §3], it follows that $\eta_{P}$ has only finitely many zeroes. Thus, Proposition 5.1.4 applies, and we have the following key result.

\section{Proposition 5.4.}

$$
\sum_{t \in \mathcal{C}} J\left(\eta_{P}, t\right)=\operatorname{deg}\left(\Omega_{\mathcal{C}}^{1} \otimes \omega^{-1}\right)=2 g-2-d .
$$

We end this section with a lemma parallel to Lemma 4.2

Lemma 5.5. For all integers $n>0$ and all points $t \in \mathcal{C}$,

$$
J\left(\eta_{P}, t\right)=J\left(\eta_{n P}, t\right) .
$$


Proof. It is clear from the local expression for $\eta$ as

$$
d w-\frac{\operatorname{Im} w}{\operatorname{Im} \tau} d \tau
$$

the $\eta_{n P}=n \eta_{P}$. The equality of local indices then follows from properties (3) and (4) of the winding number $W$.

\section{ZEROES AND INTERSECTION NUMBERS}

In this section, we relate the intersection number $I(P, t)$ to the local index $J\left(\eta_{P}, t\right)$.

Proposition 6.1. For all $t \in \mathcal{C}$

$$
J\left(\eta_{P}, t\right)=I(P, t)-1 \text {. }
$$

Proof. By Lemmas 4.2 and 5.5 we may replace $P$ with a multiple and reduce to the case where $P$ passes through the identity component of every fiber of $\mathcal{E} \rightarrow \mathcal{C}$.

Fix $t \in \mathcal{C}$ and let $\Delta_{t}$ be a neighborhood of $t$ biholomorphic to a disk and such that $\Delta_{t}^{\prime}:=\Delta_{t} \backslash\{t\}$ lies in $\mathcal{C}^{0}$. Let $z$ be a coordinate on $\Delta_{t}$ such that $t$ corresponds to $z=0$. Recall from Section 4.3 that shrinking $\Delta_{t}$ if necessary, we may lift $P$ to $\omega^{-1}$ in the exact sequence of Equation (2.2) and identify the lift with a product $w=h w_{0}$ where $w_{0}$ is a generating section of $\omega^{-1}$ (as specified in the table at the end of Section 2.3) and $h$ is a holomorphic function on $\Delta_{t}$. In terms of this data, we have

$$
\eta_{P}=d\left(h w_{0}\right)-\frac{\operatorname{Im} h w_{0}}{\operatorname{Im} \tau} d \tau
$$

The winding number that defines $J\left(\eta_{P}, t\right)$ is then $W(f)$ where

$$
f=\frac{1}{w_{0}}\left(\frac{d\left(h w_{0}\right)}{d z}-\frac{\operatorname{Im}\left(h w_{0}\right)}{\operatorname{Im} \tau} \frac{d \tau}{d z}\right) .
$$

To lighten notation, let $n=I(P, t)$, so that our goal is to prove that $W(f)=n-1$. We will complete the proof of the proposition in the next four sections, dividing into cases according to the reduction of $\mathcal{E}$ at $t$.

6.2. Points of good reduction. If $\mathcal{E}$ has good reduction at $t$, then we saw in Equation (4.1) that

$$
n:=I(P, t)=\operatorname{ord}_{z=0}(h(z)-r \tau(z)-s),
$$

where $h(t)=r \tau(t)+s$. Since $w_{0}=1$, we have $J\left(\eta_{P}, t\right)=W(f)$ where

Let

$$
f=\frac{d h}{d z}-\frac{\operatorname{Im} h}{\operatorname{Im} \tau} \frac{d \tau}{d z}
$$

$$
f_{1}(z)=\frac{d h}{d z}-r \frac{d \tau}{d z} \quad \text { and } \quad f_{2}(z)=\left(\frac{\operatorname{Im} h}{\operatorname{Im} \tau}-r\right) \frac{d \tau}{d z}
$$

Since $h-r \tau-s$ is holomorphic and vanishes to order $n \geq 1$ at $t$, we have

$$
\left|f_{1}(z)\right| \geq C_{1}|z|^{n-1}
$$

for some positive constant $C_{1}$ and all sufficiently small $z$. On the other hand,

$$
\operatorname{Im} h-r \operatorname{Im} \tau=\frac{1}{2}((h-r \tau-s)-(\overline{h-r \tau-s})),
$$


$\operatorname{Im} \tau(t)>0$, and $\tau$ is holomorphic on $\Delta$, so

$$
\left|f_{2}(z)\right| \leq C_{2}|z|^{n}
$$

for some positive constant $C_{2}$ and all sufficiently small $z$. Applying Lemma[5.1.2, we have $W(f)=$ $W\left(f_{1}\right)$, and since $f_{1}$ is holomorphic on $\Delta$ and vanishes to order $n-1$ at $z=0$, we have $W(f)=$ $W\left(f_{1}\right)=n-1$. This establishes that $J\left(\eta_{P}, t\right)=I(P, t)-1$ for all $t \in \mathcal{C}^{0}$.

6.3. Points of multiplicative reduction. Next assume that $\mathcal{E}$ has reduction type $I_{b}(b \geq 1)$ at t. According to Equation (4.2),

$$
n:=I(P, t)= \begin{cases}0 & \text { if } h(t) \notin \mathbb{R} \\ \operatorname{ord}_{z=0}(h(z)-s) & \text { if } h(t)=s \in \mathbb{R}\end{cases}
$$

and by the first part of the proof, $J\left(\eta_{P}, t\right)=W(f)$ where

$$
f=\frac{1}{w_{0}}\left(\frac{d\left(h w_{0}\right)}{d z}-\frac{\operatorname{Im}\left(h w_{0}\right)}{\operatorname{Im} \tau} \frac{d \tau}{d z}\right) .
$$

According to the table at the end of Section 2.3, $w_{0}=1$ and $\tau=(b / 2 \pi i) \log z$, so

$$
f=\frac{d h}{d z}-\frac{i \operatorname{Im} h}{z \log |z|}
$$

Suppose that $h(t) \notin \mathbb{R}$. Then after shrinking $\Delta_{t}$, we may assume that $|h(z)|$ and $|(\operatorname{Im} h(z))|$ are bounded above and below on $\Delta_{t}$ by positive constants. Since $d h / d z$ is holomorphic, we have that $|d h / d z|$ is bounded above on $\Delta_{t}$ as well. On the other hand $|1 /(z \log |z|)|>C\left|z^{-1+\epsilon}\right|$ for all $\epsilon>0$. Thus, setting $f_{1}=(i \operatorname{Im} h) /(z \log |z|)$ and $f_{2}=d h / d z$, Lemma 5.1 .2 implies that $W(f)=W\left(f_{1}\right)$. Finally,

$$
W\left(\frac{i \operatorname{Im} h}{z \log |z|}\right)=W(i \operatorname{Im} h)-W(z)-W(\log |z|)=0-1-0=-1 .
$$

Thus we find that $W(f)=-1=n-1$, establishing that $J\left(\eta_{P}, t\right)=I(P, t)-1$ when $h(t) \notin \mathbb{R}$.

To finish the multiplicative case, assume that $h(t)=s \in \mathbb{R}$. Then $|d h / d z(z)| \geq C_{1}|z|^{n-1}$ for some positive $C_{1}$ where $n=\operatorname{ord}_{z=0}(h(z)-s)$. Also, $\operatorname{Im} h \leq C_{2}|z|^{n}$, and we find that $|(i \operatorname{Im} h) /(z \log |z|)| \leq C_{2}|z|^{n-1} /|\log | z||$. Shrinking $\Delta^{\prime}$, we may assume that $C_{2}<C_{1}$ and $|(i \operatorname{Im} h) /(z \log |z|)| \leq C_{2}|z|^{n-1}$. Setting $f_{1}=d h / d z$ and $f_{2}=(i \operatorname{Im} h) /(z \log |z|)$, Lemma 5.1.2 implies that $W(f)=W\left(f_{1}\right)$. Since $f_{1}$ is holomorphic with $\operatorname{ord}_{z=0}\left(f_{1}\right)=n-1$, we conclude that $W(f)=n-1$. This establishes that $J\left(\eta_{P}, t\right)=I(P, t)-1$ when $h(t) \in \mathbb{R}$ and completes the proof for places $t$ of multiplicative reduction.

6.4. Points of potentially multiplicative reduction. Now assume that $\mathcal{E}$ has reduction type $I_{b}^{*}(b>0)$ at $t$. According to Equation (4.3),

$$
n:=I(P, t)=\operatorname{ord}_{z=0}(h(z))
$$

and by the first part of the proof, $J\left(\eta_{P}, t\right)=W(f)$ where

$$
f=\frac{1}{w_{0}}\left(\frac{d\left(h w_{0}\right)}{d z}-\frac{\operatorname{Im}\left(h w_{0}\right)}{\operatorname{Im} \tau} \frac{d \tau}{d z}\right) .
$$


According to the table at the end of Section 2.3, $w_{0}=z^{1 / 2}$ and $\tau=(b / 2 \pi i) \log z$, so

$$
f=\frac{d h}{d z}+\frac{1}{2} \frac{h}{z}-\frac{\operatorname{Im}\left(h z^{1 / 2}\right)}{z^{1 / 2}} \frac{i}{z \log |z|} .
$$

Note that

$$
\left|\frac{d h}{d z}+\frac{1}{2} \frac{h}{z}\right| \geq C_{1}|z|^{n-1}
$$

on $\Delta_{t}^{\prime}$ for some positive constant $C_{1}$. On the other hand, $\left|\operatorname{Im}\left(h z^{1 / 2}\right) / z^{1 / 2}\right| \leq C|z|^{n}$ on $\Delta_{t}^{\prime}$ for some positive constant $C$. Thus

$$
\left|\frac{\operatorname{Im}\left(h z^{1 / 2}\right)}{z^{1 / 2}} \frac{i}{z \log |z|}\right| \leq C \frac{|z|^{n-1}}{|\log | z||} .
$$

Shrinking $\Delta$, we may ensure that

$$
\left|\frac{\operatorname{Im}\left(h z^{1 / 2}\right)}{z^{1 / 2}} \frac{i}{z \log |z|}\right| \leq C_{2}|z|^{n-1}
$$

for some positive $C_{2}<C_{1}$. Setting

$$
f_{1}=\frac{d h}{d z}+\frac{1}{2} \frac{h}{z} \quad \text { and } \quad f_{2}=\frac{\operatorname{Im}\left(h z^{1 / 2}\right)}{z^{1 / 2}} \frac{i}{z \log |z|},
$$

Lemma 5.1 .2 implies that $W(f)=W\left(f_{1}\right)$, and since $f_{1}$ is holomorphic on $\Delta_{t}$ and vanishes to order $n-1$ at $z=0$, we have $W\left(f_{1}\right)=n-1$. This establishes that $J\left(\eta_{P}, t\right)=I(P, t)-1$ when $\mathcal{E}$ has reduction type $I_{b}^{*}$ at $t$, completing the proof for places $t$ of potentially multiplicative reduction.

6.5. Points of potentially good reduction. Now assume that the reduction type of $\mathcal{E}$ at $t$ is one of those of additive, potentially good reduction, namely $I_{0}^{*}, I I, I I I, I V, I V^{*} I I I^{*}$, or $I I^{*}$. According to Equation (4.3),

$$
n:=I(P, t)=\operatorname{ord}_{z=0}(h(z))
$$

and by the first part of the proof, $J\left(\eta_{P}, t\right)=W(f)$ where

$$
f=\frac{1}{w_{0}}\left(\frac{d\left(h w_{0}\right)}{d z}-\frac{\operatorname{Im}\left(h w_{0}\right)}{\operatorname{Im} \tau} \frac{d \tau}{d z}\right) .
$$

Using the table at the end of Section 2.3 , we see that $w_{0}$ is a fractional power of $z$ times a nonvanishing, holomorphic function of $\zeta$ on the domain listed in the table. From this we calculate that

$$
f_{1}:=\frac{1}{w_{0}}\left(\frac{d\left(h w_{0}\right)}{d z}\right)=\frac{d h}{d z}+\alpha \frac{h}{z}+g
$$

where $\alpha \in\{1 / 6,1 / 4,1 / 3,1 / 2,2 / 3,3 / 4,5 / 6\}$ and $g$ is holomorphic and vanishes to order $\geq n$. Thus $f_{1}$ is holomorphic on $\Delta_{t}$ and

$$
\left|f_{1}(z)\right| \geq C_{1}|z|^{n-1}
$$

for some positive constant $C_{1}$.

Now consider

$$
f_{2}=\frac{\operatorname{Im}\left(h w_{0}\right)}{w_{0} \operatorname{Im} \tau} \frac{d \tau}{d z}
$$


Since $\operatorname{ord}_{z=0}(h(z))=n$, and $\operatorname{Im} \tau$ is bounded away from zero, we find that

$$
\left|\frac{\operatorname{Im}\left(h w_{0}\right)}{w_{0} \operatorname{Im} \tau}\right| \leq C|z|^{n}
$$

on $\Delta_{t}^{\prime}$ for some positive constant $C$. On the other hand, since $\tau$ is a holomorphic function of $\zeta$, and $z=\zeta^{b}$ with $b \in\{1,3,4,6\}$, we see that

$$
\left|\frac{d \tau}{d z}(z)\right| \leq C^{\prime}|z|^{-\beta}
$$

with $\beta \in\{0,2 / 3,3 / 4,5 / 6\}$ for some positive $C^{\prime}$. It follows that

$$
\left|f_{2}(z)\right| \leq C_{2}|z|^{n-\beta}
$$

with $n-\beta>n-1$. Applying Lemma 5.1.2 we find that $W(f)=W\left(f_{1}\right)=n-1$. This establishes that $J\left(\eta_{P}, t\right)=I(P, t)-1$ when $\mathcal{E}$ has additive and potentially good reduction type at $t$.

This completes the proof of Proposition 6.1 in all cases.

\section{Proof of main theorems}

The key result of this paper is the following equality.

Theorem 7.1. Suppose that $\pi: \mathcal{E} \rightarrow \mathcal{C}$ is non-constant and that $P$ is a section of $\pi$ of infinite order. Let $g$ be the genus of $\mathcal{C}$ and let $d$ be the degree of the line bundle $\omega=O^{*}\left(\Omega_{\mathcal{E} / \mathcal{C}}^{1}\right)$. Let $I(P, t)$ be the local intersection indices defined in Section 4.1. Then

$$
\sum_{t \in \mathcal{C}}(I(P, t)-1)=2 g-2-d .
$$

Proof. Let $\eta_{P}$ be the 1-form attached to $P$ in Section 5.3. Then according to Proposition 5.4 we have

$$
2 g-2-d=\sum_{t \in \mathcal{C}} J\left(\eta_{P}, t\right)
$$

and according to Proposition 6.1

$$
J\left(\eta_{P}, t\right)=I(P, t)-1
$$

for all $t \in \mathcal{C}$.

Corollary 7.2. Let $T_{\text {Betti }}$ be the set of points $t \in \mathcal{C}$ where $I(P, t) \geq 2$. Then

$$
\left|T_{\text {tor }}\right| \leq\left|T_{\text {Betti }}\right| \leq 2 g-2-d+\delta
$$

where $\delta$ is the number of singular fibers of $\pi: \mathcal{E} \rightarrow \mathcal{C}$.

Proof. From the definitions, $T_{\text {tor }}$ is the subset of $T_{\text {Bett }}$ where $P(t)$ is a torsion point in its fiber, so $\left|T_{\text {tor }}\right| \leq\left|T_{\text {Betti }}\right|$. Let $S$ be the set of points of $\mathcal{C}$ where $\mathcal{E}$ has bad reduction. Then $I(P, t) \geq 0$ for all $t \in \mathcal{C}, I(P, t) \geq 1$ for all $t \notin S$, and $I(P, t) \geq 2$ if and only $t \in T_{\text {Betti }}$. Thus by the Theorem,

$$
2 g-2-d \geq\left|T_{\text {Betti }}\right|-|S|
$$

and the corollary follows immediately. 


\section{EXAMPLES AND APPLICATIONS}

We consider some explicit families illustrating various aspects of the main theorem, and we give an application to bounding heights of integral points on elliptic curves over function fields.

Suppose as usual that $\pi: \mathcal{E} \rightarrow \mathcal{C}$ is a Jacobian elliptic surface with zero section $O, g$ is the genus of $\mathcal{C}, d=\operatorname{deg}\left(O^{*}\left(\Omega_{\mathcal{E} / \mathcal{C}}^{1}\right)\right)$, and $\delta$ is the number of singular fibers of $\pi$.

Proposition 8.1. If $2 g-2-d+\delta<0$, then the group of sections of $\pi$ is finite.

Proof. Suppose $P$ is a section of $\pi$ of infinite order, and let $T_{\text {tor }}$ be the corresponding set of tangencies between $P$ and torsion multisections. Then by Corollary 7.2 the cardinality of $T_{\text {tor }}$ would be negative, a contradiction. Thus, there are no sections of infinite order.

It would be interesting to have a more direct proof of the proposition. We note that the proposition is sharp in the sense that we give examples below of elliptic surfaces with $2 g-2-d+\delta=0$ and with a section of infinite order.

8.2. Degenerate cases. Next, we give two examples where $2 g-2-d+\delta<0$, one isotrivial, one non-isotrivial. In both cases, it is straightforward to check that the group of sections is torsion, in agreement with Proposition 8.1 .

Let $E$ be any elliptic curve over $\mathbb{C}$ with a given Weierstrass model $y^{2}=x^{3}+a x+b$ where $a, b \in \mathbb{C}$. Let $E$ be the twisted elliptic curve

$$
E: \quad y^{2}=x^{3}+a t^{2} x+b t^{3}
$$

over $\mathbb{C}(t)$, and let $\mathcal{E} \rightarrow \mathbb{P}^{1}$ be the regular minimal model of $E / \mathbb{C}(t)$. Then one verifies easily that $d=1$ and $\delta=2$ ( $E$ has $I_{0}^{*}$ reduction at $t=0$ and $t=\infty$ and good reduction elsewhere), so that $2 g-2-d+\delta=-1$.

For a non-isotrivial example, consider

$$
E: \quad y^{2}=x^{3}-3 t^{4}\left(t^{2}-1\right)^{2} x+2 t^{5}\left(t^{2}-1\right)^{3}
$$

over $\mathbb{C}(t)$, and let $\mathcal{E} \rightarrow \mathbb{P}^{1}$ be the regular minimal model. Then one verifies that $d=2$ and $\delta=3$, so $2 g-2-d+\delta=-1$. Moreover, the $j$-invariant of $E$ is $1728 t^{2} /\left(t^{2}-1\right)$, so $\mathcal{E} \rightarrow \mathbb{P}^{1}$ is nonisotrivial. (Thanks to Rick Miranda for pointing out how to construct an example like this.) We refer to [SH85] and [Ngu99] for the complete list of Jacobian elliptic surfaces over $\mathbb{P}^{1}$ with three singular fibers.

We have the following general result.

Proposition 8.3. Suppose that $\pi: \mathcal{E} \rightarrow \mathcal{C}$ is a facobian elliptic fibration with zero section O. Suppose $\pi$ has everywhere semi-stable reduction (i.e., the bad fibers are of type $I_{b}$ ) and is non-isotrivial. Then

$$
2 g-2-d+\delta>0 .
$$

Here, as usual, $g$ is the genus ofC $d=\operatorname{deg}\left(O^{*}\left(\Omega_{\mathcal{E} / \mathcal{C}}^{1}\right)\right)$, and $\delta$ is the number of singular fibers of $\pi$.

Proof. Let $D$ be the divisor

$$
D=O+\sum_{\operatorname{bad} t} \pi^{-1}(t)
$$


where the sum is over the set of points where the fiber of $\pi$ is singular. By hypothesis, each $\pi^{-1}(t)$ appearing in the sum is a chain of $\mathbb{P}^{1} \mathrm{~s}$ meeting in nodes, and the divisor $D$ thus has normal crossings.

Consider the logarithmic Chern classes $\bar{c}_{1}(\mathcal{E}, D)$ and $\bar{c}_{2}(\mathcal{E}, D)$ as in [Urz11]. By [Urz11, Thm. 9.2] (which applies since $\pi$ is non-isotrivial), we have

$$
\bar{c}_{1}(\mathcal{E}, D)^{2}<3 \bar{c}_{2}(\mathcal{E}, D) .
$$

From the definitions, one computes that

$$
\bar{c}_{1}(\mathcal{E}, D)^{2}=4 g-4+d+2 \delta \quad \text { and } \quad \bar{c}_{2}(\mathcal{E}, D)=2 g-2+\delta,
$$

so we find that

$$
0<3 \bar{c}_{2}(\mathcal{E}, D)-\bar{c}_{1}(\mathcal{E}, D)^{2}=2 g-2-d+\delta
$$

as desired.

8.4. Optimality in the constant case. Fix an elliptic curve $E$ over $\mathbb{C}$ and a positive integer $g$. Let $B=2 g-2$ and let $A$ be any integer with $0 \leq A \leq B$. We will produce a constant elliptic surface $\mathcal{E}=\mathcal{C} \times E$ and a section whose corresponding $T_{\text {tor }}$ satisfies

$$
\left|T_{\text {tor }}\right|=A \leq B=2 g-2 .
$$

Indeed, by the Riemann existence theorem, there exists a branched cover $f: \mathcal{C} \rightarrow \mathcal{E}$ where $\mathcal{C}$ is a curve of genus $g$ and $f$ has simple ramification. I.e., $f$ is ramified over $2 g-2$ points with distinct images in $\mathcal{C}$, and the ramification indices are all 2. Moreover, we may choose the points in $E$ where $f$ is ramified freely.

Let $\mathcal{E}=\mathcal{C} \times E$ and let $P$ be the section corresponding to the map $f$. Then $T_{\text {Bett } i}$ is exactly the set of branch points, and it has cardinality $2 g-2$. Moreover, by suitable choice of those points, we may arrange for $\left|T_{\text {tor }}\right|$ to take any value between 0 and $2 g-2$. This shows that Corollary 7.2 is sharp in the constant case.

8.5. Optimality in the non-constant cases. A similar idea works in the non-constant cases once we have suitable starting data. To that end, we construct $\pi: \mathcal{E}_{1} \rightarrow \mathbb{P}^{1}$ with

$$
2 g-2-d+\delta=-2-d+\delta=0,
$$

and with a section $P_{1}$ of infinite order. By Corollary 7.2 there are no tangencies bewteen $P_{1}$ and a torsion multisection.

In the isotrivial case, we may take the example considered in [UU19, §7], namely the quotient of the square of an elliptic curve by the diagonal map $(t, t) \mapsto(-t,-t)$. The minimal regular model $\mathcal{E}_{1} \rightarrow \mathbb{P}^{1}$ has $d=2$ and $\delta=4$ bad fibers and a section $P_{1}$ of infinite order (namely the image of the graph of the identity map). The set of tangencies between $P_{1}$ is thus empty by Corollary 7.2

For a non-isotrivial example, consider the elliptic curve

$$
E_{1}: \quad y^{2}=x^{3}-t x+t
$$

and let $\mathcal{E}_{1} \rightarrow \mathbb{P}^{1}$ be the regular minimal model. One computes that $\mathcal{E}_{1}$ has $\operatorname{deg}\left(O^{*}\left(\Omega_{\mathcal{E}_{1} / \mathbb{P}^{1}}^{1}\right)\right)=1$, and good reduction away from $t=0, t=27 / 4$, and $t=\infty$, and has bad reduction at these points. Thus for any non-torsion section, the corresponding set of torsion tangencies $T_{\text {tor }}$ is empty. Let $P_{1}$ be the section corresponding to the rational point $(x, y)=(1,1)$. Straightforward 
calculation shows that $P_{1}$ is of infinite order (and in fact generates the group of sections of $\mathcal{E}_{1}$ ). By Corollary 7.2 , the corresponding et $T_{\text {tor }}$ is empty.

Now fix a positive, even integer $B$ and let $f: \mathcal{C} \rightarrow \mathbb{P}^{1}$ be a branched cover with exactly $B$ ramification points and simple branching over each one. (We could also insist that $\mathcal{C} \rightarrow \mathbb{P}^{1}$ have low degree, say degree 2, but this is not relevant for what follows.) Let $\mathcal{E} \rightarrow \mathcal{C}$ be the regular minimal model of the pull back of $\mathcal{E}_{1} \rightarrow \mathbb{P}^{1}$ to $\mathcal{C}$ (where $\mathcal{E}_{1}$ is either of the examples above), and let $P$ be the section induced by $P_{1}$. Assuming that the branch points of $\mathcal{C} \rightarrow \mathbb{P}^{1}$ are distinct from the points where $\mathcal{E}_{1}$ has bad reduction, we have $I(P, t)=e_{f}(t)$ where $e_{f}(t)$ is the ramification index of $f$ at $t \in \mathcal{C}$.

The Riemann-Hurwitz formula yields

$$
2 g_{\mathcal{C}}-2-d+\delta=B
$$

where $d$ and $\delta$ are the usual invariants attached to $\mathcal{E}$. Thus, Corollary 7.2 implies that $\left|T_{\text {tor }}\right| \leq B$.

By choosing some of the branch points of $f$ to be among the points of $\mathbb{P}^{1}$ where $P$ takes a torsion value, we may arrange for $\left|T_{\text {tor }}\right|$ to take any value between 0 and $B$. This shows that Corollary 7.2 is sharp.

8.6. Height bounds. We consider bounds on heights of integral points over function fields, as pioneered by Mason [Mas84 Th. 12, p. 58]. Our bound is a small improvement over that of Hindry and Silverman [HS88, Props. 8.2 and 8.3]. Although we work over $\mathbb{C}$, the generalization to any field of characteristic zero is straightforward.

To state the result, let $\mathcal{C}$ be an irreducible, smooth, projective curve of genus $g$ over $\mathbb{C}$, let $K$ be the function field $K=\mathbb{C}(\mathcal{C})$, let $E$ be an elliptic curve over $K$, and let $\hat{h}: E(K) \rightarrow \mathbb{Q}$ be the canonical height function on $E$. (We give a precise definition in the proof below.)

Let $S$ be a non-empty, finite set of closed points of $\mathcal{C}$, and let $\mathcal{O} \subset K$ be the subring of functions regular at all points not in $S$. Choose an $S$-integral model for $E$, in other words, a Weierstrass model

$$
y^{2}=x^{3}+A x+B
$$

where $A, B \in \mathcal{O}$. Let $\Delta=4 A^{3}+27 B^{2}$ be the discriminant of this model, and let $T$ be the union of $S$ and the set of points where $\Delta$ vanishes. We write $|T|$ for the cardinality of $T$.

Theorem 8.7. Suppose that $P \in E(K)$ is a non-torsion point that is $S$-integral, i.e., whose coordinates $x(P)$ and $y(P)$ in the model (8.1) are in $\mathcal{O}$. Then we have

$$
\hat{h}(P) \leq 4 g-4+2|T| .
$$

Proof. Let $\pi: \mathcal{E} \rightarrow \mathcal{C}$ be the Néron model of $E / K$, and write $O$ and $P$ for the images in $\mathcal{E}$ of the zero-section and the section induced by $P$ respectively.

We first recall the definition of $\hat{h}(P)$ as an intersection number following [CZ79] and [Shi90]. Associated to $P$ there is a unique $\mathbb{Q}$-linear combination of non-identity components of fibers of $\pi$ denoted $D_{P}$ with the property that

$$
\left(P-O+D_{P}\right) \cdot C=0
$$

for every component $C$ of every fiber of $\pi$. (The dot signifies the intersection pairing on $\mathcal{E}$.) The canonical height is then

$$
\hat{h}(P)=-\left(P-O+D_{P}\right) \cdot(P-O) \in \mathbb{Q} .
$$


Consulting the table [CZ79, 1.19] reveals that the coefficients of $D_{P}$ are non-negative, and the canonical bundle formula for $\mathcal{E}$ and adjunction show that $O . O=P . P=-d$ where $d=\operatorname{deg}(\omega)$ is as in the introduction. Thus we find

$$
\hat{h}(P) \leq-(P-O) \cdot(P-O)=2(P . O)+2 d .
$$

To finish the proof, we will estimate $P . O$ using Theorem 7.1. Since $P \neq O$, the intersection number P.O is a sum of local terms, and we write $(P . O)_{t}$ for the contribution at points of intersection lying in $\pi^{-1}(t)$. If $t \notin T$, the model (8.1) is minimal and has good reduction, and $x(P)$ and $y(P)$ are regular (i.e., do not have poles), so we have $(P . O)_{t}=0$. Also, $I(P, t)-1 \geq 0$ at all points of good reduction. Thus

$$
\sum_{t \notin T}(P . O)_{t} \leq \sum_{t \notin T}(I(P, t)-1) .
$$

For any $t$, we have $(P . O)_{t} \leq I(P, t)$, so we also have

$$
\sum_{t \in T}(O . P)_{t} \leq \sum_{t \in T} I(P, t)=\sum_{t \in T}(I(P, t)-1)+|T| .
$$

Adding the last two displayed equations and applying Theorem 7.1 yields

$$
P . O \leq \sum_{t \in \mathcal{C}}(I(P, t)-1)+|T|=2 g-2-d+|T| .
$$

Using this in Equation (8.2) yields the theorem.

Remark 8.8. From the proof, we see that the inequality of the theorem is an equality if and only if (i) $P$ passes through the identity component of every bad fiber (so $D_{P}=0$ and there are no "correction terms"); and (ii) $P . O=2 g-2-d+|T|$. We give two examples where these conditions are satisfied, thus showing that the theorem is sharp in these cases.

For an isotivial example, take the curve $E$ over $\mathbb{C}(t)=\mathbb{C}\left(\mathbb{P}^{1}\right)$ considered in [UU19, §7] and in Section 8.5 above (associated to $E_{0}$ given by $y^{2}=f(x)=x^{3}+a x+b$ ) and the point $P_{2}$ induced by multiplication by 2 on $E_{0}$. Let $S=\{\infty\}$. Then the model

$$
y^{2}=x^{3}+a f(t)^{2}+b f(t)^{3}
$$

is $S$-integral and the set $T$ consists of $S$ and the three roots of $f$ and has cardinality 4 . One checks that $P_{2}$ is $S$-integral, the corresponding section passes through the identity component of every fiber, and $P . O=0=2 g-2-d+|T|$.

For a non-isotrivial example, take the other example considered in Section 8.5, namely

$$
E_{1}: \quad y^{2}=x^{3}-t x+t
$$

over $\mathbb{C}(t)$ and let

$$
P=2(1,1)=\left(\frac{1}{4} t^{2}-\frac{3}{2} t+\frac{1}{4}, \frac{1}{8} t^{3}-\frac{9}{8} t^{2}+\frac{15}{8} t+\frac{1}{8}\right) .
$$

If $S=\{\infty\}$, then this model and the point $P$ are $S$-integral and the corresponding set $T$ is $\{0,27 / 4, \infty\}$. Again one finds that the section corresponding to $P$ passes through the identity component of every fiber, and P.O $=0=2 g-2-d+|T|$. 


\section{REFERENCES}

[ACZ18] Y. André, P. Corvaja, and U. Zannier, The Betti map associated to a section of an abelian scheme, 2018. Preprint, arXiv:1802.03204.

[BT82] R. Bott and L. W. Tu, Differential forms in algebraic topology, Graduate Texts in Mathematics, vol. 82, Springer-Verlag, New York-Berlin, 1982.

[CDMZ19] P. Corvaja, J. Demeio, D. Masser, and U. Zannier, On the torsion values for sections of an elliptic scheme, 2019. Preprint, arxiv:1909.01253.

[CMZ18] P. Corvaja, D. Masser, and U. Zannier, Torsion hypersurfaces on abelian schemes and Betti coordinates, Math. Ann. 371 (2018), 1013-1045.

[CZ79] D. A. Cox and S. Zucker, Intersection numbers of sections of elliptic surfaces, Invent. Math. 53 (1979), 1-44.

[Ful95] W. Fulton, Algebraic topology, Graduate Texts in Mathematics, vol. 153, Springer-Verlag, New York, 1995. A first course.

[HS88] M. Hindry and J. H. Silverman, The canonical height and integral points on elliptic curves, Invent. Math. 93 (1988), 419-450.

[Kod63a] K. Kodaira, On compact analytic surfaces. II, Ann. of Math. (2) 77 (1963), 563-626.

[Kod63b] _ On compact analytic surfaces. III, Ann. of Math. (2) 78 (1963), 1-40.

[Mas84] R. C. Mason, Diophantine equations over function fields, London Mathematical Society Lecture Note Series, vol. 96, Cambridge University Press, Cambridge, 1984.

[Ngu99] K.-V. Nguyen, Extremal elliptic fibrations and singular K3 surfaces, Tokyo J. Math. 22 (1999), 415-424.

[SH85] U. Schmickler-Hirzebruch, Elliptische Flächen über $\mathrm{P}_{1} \mathbf{C}$ mit drei Ausnahmefasern und die hypergeometrische Differentialgleichung, Schriftenreihe des Mathematischen Instituts der Universität Münster, 2. Serie [Series of the Mathematical Institute of the University of Münster, Series 2], vol. 33, Universität Münster, Mathematisches Institut, Münster, 1985.

[Shi90] T. Shioda, On the Mordell-Weil lattices, Comment. Math. Univ. St. Paul. 39 (1990), 211-240.

[Urz11] G. Urzúa, Arrangements of rational sections over curves and the varieties they define, Atti Accad. Naz. Lincei Rend. Lincei Mat. Appl. 22 (2011), 453-486.

[UU19] D. Ulmer and G. Urzúa, Transversality of sections on elliptic surfaces with applications to elliptic divisibility sequences and geography of surfaces, 2019. Preprint, arXiv:1908.02208.

Department of Mathematics, University of Arizona, Tucson, AZ 85721 USA

E-mail address: ulmer@math. arizona.edu

Facultad de Matemáticas, Pontificia Universidad Católica de Chile, Santiago, Chile

E-mail address: urzua@mat.uc.cl 